\title{
Technique pour technique, une médiation : la traduction
}

Sylvie Landois-Maynard

\section{CpenEdition}

\section{Journals}

Édition électronique

URL : http://journals.openedition.org/asp/4260

DOI : $10.4000 /$ asp. 4260

ISSN : 2108-6354

\section{Éditeur}

Groupe d'étude et de recherche en anglais de spécialité

\section{Édition imprimée}

Date de publication : 1 octobre 1993

Pagination : $11-28$

ISSN : 1246-8185

\section{Référence électronique}

Sylvie Landois-Maynard, «Technique pour technique, une médiation : la traduction », ASp [En ligne],

2 | 1993, mis en ligne le 22 février 2014, consulté le 01 mai 2019. URL : http://journals.openedition.org/ asp/4260 ; DOl : 10.4000/asp.4260

Ce document a été généré automatiquement le 1 mai 2019.

Tous droits réservés 


\title{
Technique pour technique, une médiation : la traduction
}

\author{
Sylvie Landois-Maynard
}

1 «La traduction touche à la fois au monde universitaire et professionnel. Elle offre aux étudiants que nous formons un produit authentique, un service véritable » (LandoisMaynard 1009: 47).

2 En démonstration de cette proposition, voici la mise à profit de la confrontation de deux domaines: la spécialité technique et la langue seconde de spécialité, par le biais de la pratique raisonnée de la traduction ${ }^{1}$, qui joue un rôle médiateur, de spécialité à spécialité.

3 Le cadre de l'enseignement se situe au département informatique de l'Institut Universitaire de Technologie de l'Université René Descartes, en cycle initial et en formation continue. L'expérience se poursuit lors d'un séminaire annuel sur la traduction, au département Techniques de Commercialisation, en DUTA ${ }^{2}$; se prolonge dans une formation Langue, Thème, Version, en IUFM à l'UFR de Linguistique générale et appliquée, à l'Université Paris 5 ; enfin, se complète d'une correction de traductions en anglais de l'informatique, en BTS de $1^{\text {re }}$ et $2^{\mathrm{e}}$ années, au CNED de Poitiers/Futuroscope.

4 Les qualifications de nos étudiants sont inversement proportionnelles. Il faut distinguer les étudiants de formation initiale, qui débutent une formation dans la spécialité, et ont une connaissance de l'anglais général, du niveau baccalauréat ou au-delà, et les étudiants de formation continue, qui sont déjà des professionnels, mais pour qui la connaissance de l'anglais est souvent empirique et passe, en grande partie, par l'écrit technique (consultation de documents et instructions en langue anglaise).

5 Il ne s'agit pas d'imposer la traduction, ou de la greffer, d'une manière parasitaire, sur l'enseignement de l'anglais. Il convient au contraire de faire de la traduction « un facilitateur de passage ", un élément moteur qui contribue à l'étude spécifique de la langue de spécialité, au sein d'une formation dans le domaine de spécialité. Tout en conservant l'optique de la compréhension orale et écrite, de l'expression orale et écrite, on ne pratique pas un genre nouveau, mais on renouvelle une pratique de la traduction, 
longtemps considérée comme le parent pauvre dans l'enseignement des langues. Mieux encore, on en fait un catalyseur de cet enseignement.

Nous développerons un exemple en particulier et quelques stratégies dont nous avons éprouvé le réalisme à l'usage. Le travail se construit sur la base d'une confrontation négociée, au point de confluence de deux domaines de spécialité : dans le cas présent, l'informatique et la langue anglaise. Le champ est suffisamment ouvert pour aborder des thèmes qui intéressent toute autre spécialité.

7 Nous entreverrons une perspective vers une systématisation possible d'un tel enseignement, où la traduction, dosée et harmonieusement intégrée au cours, démontre des qualités spécifiques dans la pédagogie d'acquisition de la langue, et des vertus médiatiques propres à toute communication.

\section{Traduction, outil ou fin en soi ?}

8 La traduction peut être systématique et correspondre à un sujet enseigné, à l'exemple du thème et de la version universitaires. À l'opposé, le refus catégorique de traduire fait partie d'un principe pédagogique qui trouve ses défenseurs.

9 Sur une quinzaine d'années, les instructions officielles, en particulier dans l'enseignement du second degré, ont tout d'abord proscrit la traduction, puis l'ont admise à usage de tester les acquis lexicaux, et de réutiliser les structures grammaticales.

Or, promotion après promotion, sans qu'on mentionne la traduction ou s'y réfère, les étudiants "traduisent » spontanément, souvent pour eux-mêmes et, si quelque aspect vient à leur manquer, ils exposent à l'occasion leurs difficultés.

11 Si on leur demande raison de leur passage à la traduction, ils disent qu'ils sont obligés de " passer », ou de « repasser », par leur langue maternelle 3 , pour « aller » vers l'anglais. Ils évoquent le besoin de comprendre parfaitement le texte, écrit ou oral, proposé. Cette compréhension " transite ", assez naturellement par la traduction.

12 Celle-ci, dans les conditions optimales, devrait assurer une compréhension du texte écrit ou oral, au plus haut degré, à savoir la maîtrise de l'aspect technique du document, et la maitrise de la langue qui véhicule cette technicité.

13 Si l'on fait le décompte des diverses implications contenues dans ces deux "maîtrises ", on obtient, entre autres ${ }^{4}$, l'apprentissage de la connaissance :

- technique du domaine de spécialité,

- de la langue anglaise de spécialité,

- de la langue anglaise proprement dite,

- de la langue française de spécialité,

- de la langue française proprement dite (langue maternelle ou acquise),

- de la maîtrise du passage d'une langue à l'autre, dans les deux sens,

- de la maîtrise de ce passage dans le domaine de spécialité.

On doit y ajouter la certitude de véritablement «traduire/translater» le document de base dans une autre langue, même si cette démarche se fait par plusieurs paliers, par réajustements successifs, d'une manière dynamique et évolutive; la possibilité de communiquer un produit quasiment fini, dont on arrête le choix à partir de certains critères de communicabilité. 

peut énumérer les points suivants : le partage de l'information, tant dans le domaine de spécialité, que dans celui de la langue qu'il faut maîtriser, donne lieu à un échange de vocabulaire technique dans les deux langues ; à une connaissance de la phrase en langue seconde, qui utilise ce vocabulaire spécifique et sert de support à la démonstration technique; à un décodage, et à un encodage, des mécanismes et des démonstrations contenues dans le texte de spécialité ; ceci dans l'une et l'autre langue.

Il s'agit encore de prévoir une confrontation des productions individuelles ou collectives, pour en tester la pertinence et le bien-fondé ; puis d'opérer les ajustements, les retouches et les corrections nécessaires. anticiper ; ensuite, justifier et systématiser ce que l'on pratique ; enfin, le tenir au nombre des acquis théoriques qui permettront le réemploi.

L'étudiant entre là dans un domaine d'une riche complexité ; mais il en prend conscience au fil de sa formation, qui est en partie sous la responsabilité de notre enseignement. Sa participation et son implication activent découvertes et réflexions.

19 Sans minimiser le rôle de la traduction "optionnelle », qui teste les acquis, et aide au réemploi des structures, la traduction peut systématiquement prendre trois créneaux :

- elle sert de tremplin à la compréhension d'un support, écrit ou oral (texte, énoncé, article, résumé de vidéo...) ;

- en position médiane, en parcours de support, et en manière de commentaire, elle opère les transitions nécessaires, en autant de points choisis ;

- en position finale, elle assure un produit, plus ou moins fini, le résultat d'un commentaire qu'elle parachève.

Ces trois positions clés de la traduction correspondent à trois degrés différents de maitrise. Comme tremplin ou levier pour s'assurer du sens, donc en tant qu'outil de production, elle nécessite un travail d'apprenant, voire d'apprenti. Lorsqu'elle est médiatique et transitionnelle, elle recouvre le travail de l'enseignant, du tuteur. Comme produit élaboré ou finalité, elle devient le travail du spécialiste, du professionnel.

21 Ce sont sur ces trois parcours, dans un va-et-vient de l'un à l'autre, que l'étudiant va négocier la traduction, avec une maitrise et une autonomie plus ou moins grandes, par étapes plus ou moins progressives.

\section{Traduction en interactivité}

La traduction participe de l'enseignement des langues, c'est un instrument pédagogique et un facteur de communication de toute importance : elle favorise les passages d'une langue à l'autre, elle utilise les techniques médiatiques, le matériel adapté à ces techniques. Elle est elle-même communicante et produit de communication. La maîtriser, c'est aussi connaître et éprouver tous les outils des multimédias. Dans le groupe classe, cela implique de constituer une équipe solide, aux partages nombreux aux tâches bien définies et réparties harmonieusement.

Les espaces de formation sont ceux de l'enseignement des langues : le plan de la classe, le laboratoire, la salle informatique, le studio. Les "moments" de formation sont les mêmes : cours, réunion, séminaire, conférence, téléconférence. Le matériel est identique : 
magnétophone, magnétoscope, télévision, ordinateur, console. Les supports, aussi : texte, image, discours...

Les techniques pédagogiques utilisées dans la traduction, sont celles de l'enseignement des langues : passage de l'oral à l'écrit, de l'écrit à l'oral, lexique général et lexique de spécialité, morphosyntaxe, grammaire, exercices de reconnaissance et de réemploi, progression, évaluation...

Le créneau spécifique à la traduction se partage sur la traduction proprement dite et l'interprétation, ceci dans le domaine de spécialité. Il est vrai que le domaine propre à la traduction déborde largement le cadre de la formation, et s'ouvre vers le professionnel, une étape que certains de nos étudiants ont déjà franchie, ou franchiront.

La "passerelle » la plus intéressante à exploiter, dans le cadre de la formation en IUT, reste le lien entre l'apprentissage de l'anglais et celui de la spécialité technique (informatique, gestion, commercialisation, publicité, relations publiques, chimie, mesures physiques, médecine...). Ce lien se vit d'une façon optimale dans l'espace-groupe, où les idées de chacun sont brassées et testées, et où le travail est supervisé, de manière professionnelle, par des spécialistes.

27 Le travail du groupe est le reflet du travail individuel; mais dans le groupe, les tâches « objectivées » rendent possibles les commentaires. La réflexion est mise en commun, au profit de tous. Par paire, par équipe, semi-groupe, groupe ou classe entière, la production individuelle s'étoffe et s'enrichit. On met en place un entraînement authentique, par conséquent une formation.

Les centres d'intérêt sont délimités, l'itinéraire est défini et on prévoit des aides sur le parcours. Le travail de chaque équipe est rigoureusement déterminé. Les équipes sont cependant modulables et interchangeables, de projet en projet.

Nous prendrons pour exemple, un projet de traduction de publicité en langue anglaise (annexe 1), sur un produit informatique. C'est un terrain d'apprentissage privilégié5. En effet, le domaine publicitaire offre texte, image et discours.

L'aspect culturel, la communication vers un public ciblé, l'évolution historique des produits, l'évolution technique, le monde de la recherche, sont autant de domaines à explorer. La typographie, la mise en page, la présentation pour des publics différents, dans des langues différentes, représentent autant de secteurs à exploiter.

Le langage, spécifiquement technique, y est d'une rigueur remarquable, puisque l'espace est réduit et optimisé. La mise en valeur, dans le texte, des qualités techniques du produit, implique un vocabulaire en langue générale, spécifique à la communication, démonstratif et convaincant.

Le travail est réparti de la manière suivante :

\section{Équipe 1}

$1^{r e}$ approche $=$ description en langue anglaise ${ }^{6}$ du produit publicitaire : présentation, typologie, emplacement des illustrations, fiche technique.

$2^{e}$ approche $=$ discussion en anglais sur les différences de réutilisation de l'encart publicitaire, pour le public français: public/cible, choix d'une présentation éventuellement différente, mise en page de l'encart technique, des schémas, des photos. Préparation d'une maquette.

Équipe 2 
pproche = étude du lexique général, du jargon publicitaire, constitution des phrases. Le vocabulaire est mis en catégories. Commentaire sur l'aspect culturel et commercial. Travail sur les niveaux de langue (usuelle, vulgarisation scientifique, démonstration...). Ton du texte (humoristique, emphatique, accroche commerciale...).

$2^{e}$ approche = traduction de ce lexique en français. Travail dans le contexte, puisque la traduction passe nécessairement par la compréhension et le commentaire.

Équipe 3 document, ou bien «à l'inverse » de la langue utilisée dans le document. Références historiques, géographiques, sociologiques, cartes, graphes, données » chiffrées récentes. On fait appel ici à toute référence culturelle, ou à toute connaissance spécifique (lectures de revues spécialisées, stages en entreprise, expérience professionnelle, études en parallèle, séjours à l'étranger). une mise au point à double entrée, puisqu'ils évaluent le commentaire apporté, et donnent leur point de vue sur la traduction.

(bilan, évaluation, méthodologie, mise en théorie) portent sur chaque créneau (présentation du produit de publicité, étude du lexique, démonstration documentée), puisque la traduction a accompagné un bon nombre d'exercices. Seront étudiés en particulier: la pertinence lexicale en contexte, la cohérence de l'information, la justesse du ton dans l'échange en référence au culturel, l'intonation, et le rythme caractérisant chaque langue. Ces démarches une fois faites, reste la traduction de la page publicitaire, proprement dite. Le travail déjà fourni a posé 
les fondements. La traduction en est donc facilitée. Les quatre équipes, préparées précédemment, riches des matériaux mis en commun, se mettent au travail.

L'interprétation précède la traduction. On en conserve la trace sur cassette audio ou vidéo. La vidéo concrétise efficacement la mise en situation (trace visuelle des mots sur les lèvres, conviction des regards, mimiques, comportement...). Si l'on travaille à partir des cassettes d'enregistrement, on prévoit sur la bande-son, des plages de silence conséquentes, pour laisser suffisamment d'espace sonore et renégocier son interprétation 9 .

49 À l'écrit, il est pratique d'adopter des feuilles transparentes ou suffisamment d'interlignes, pour prévoir différentes adaptations, autant de brouillons ou d'esquisses de traduction. Chaque étudiant prend ainsi conscience d'une traduction évolutive, en voie de perfectionnement.

On travaille délibérément les nuances, et par conséquent, toutes les cristallisations possibles à partir d'un mot, d'une phrase ou d'un ensemble de phrases, autour d'une idée. Les commentaires, de l'équipe au groupe, prennent là toute leur valeur et leur cohérence.

51 Le travail peut être démultiplié selon le temps dont on dispose ${ }^{10}$. Les étudiants travaillent individuellement sur une partie, ou la totalité de la traduction, mettent leur travail en parallèle par paires, puis en commun par équipes. Les équipes travaillent alors au sein du groupe, enfin les groupes en classe entière. Les difficultés trouvent leur solution, à l'un et l'autre niveau.

52 Il y a, tout au long, une authentique négociation, avec contradiction et justification, voire auto-évaluation. Le texte publicitaire a été largement préparé et commenté, pour déboucher sur une traduction rigoureuse et fluide.

Le commentaire porte désormais sur la traduction du document, et démontre la pertinence de la préparation de base, puisque la traduction utilise, à profit, la rigueur langagière et technique du travail précédent. De plus, elle débouche sur des perspectives exploitables, puisqu'elle communique une page ou un discours techniques, en langue d'arrivée, ceci pour un public potentiel. Dans le meilleur des cas, le produit traduit est de qualité quasi professionnelle. On peut ne pas atteindre à un tel niveau, ne faire qu'une partie de l'itinéraire, marquer des paliers et limiter le travail à telle ou telle séquence (annexe 2).

\section{«Processus-tiers »}

54 Ce travail effectué est de toute importance pour l'étudiant en anglais de spécialité. Il s'aperçoit qu'il a besoin, non seulement d'être technicien dans son domaine (informatique, génie électrique, génie thermique...), mais qu'il doit progresser dans le créneau que lui offre l'acquisition de la langue de spécialité. Cette dernière fait partie de son bagage professionnel et il en fera vite l'expérience.

Il lui faut maîtriser les techniques langagières, à la fois de sa langue maternelle et de la langue acquise. Cette langue seconde sera, à l'exemple de sa langue naturelle, à double volet: langue spécifique à une technique, d'une part; d'autre part, moyen de communication de cette technique et, au-delà, moyen de communiquer tout simplement. 

recul nécessaire à cette réflexion. L'expérience des groupes précédents s'avère utile, et garder une trace écrite (manuscrit ou traitement de texte...) et orale (film, cassette...), permet un travail sur le long terme. Des horizons s'ouvrent, les étudiants constatent, à l'évidence, qu'ils sont capables de mener à bien, à leur niveau dans la spécialité, dans la langue aussi bien que dans la technique, une tâche reconnue difficile, soit d'interprétation, soit de traduction. traducteurs et interprètes, qui apporteront une expérience authentique en matière de traduction; pratiquer des ouvertures vers le monde professionnel de la traduction de spécialité, et trouver accès aux salles de conférences internationales; faciliter la prise de 
conscience et la réflexion sur le travail en cours; proposer, autant qu'il est possible, un projet qui aboutisse et soit authentique.

64 élitiste, la traduction est une ouverture à la communication, et fait partie de l'expérience
quotidienne à l'internationale. L'intérêt restreint, voire inexistant qu'on lui réservait, doit s'élargir à une curiosité fructueuse.

Le domaine technique et l'anglais de spécialité s'enrichissent mutuellement; leur confrontation est fertile. La traduction est, à leur point de confluence, une médiation véritable.

\section{BIBLIOGRAPHIE}

Landois, Sylvie. 1990. « La traduction coordonnée ». Les Cahiers de l'APLIUT 10/1, 32-47.

\section{ANNEXES}

\section{Annexe 1}

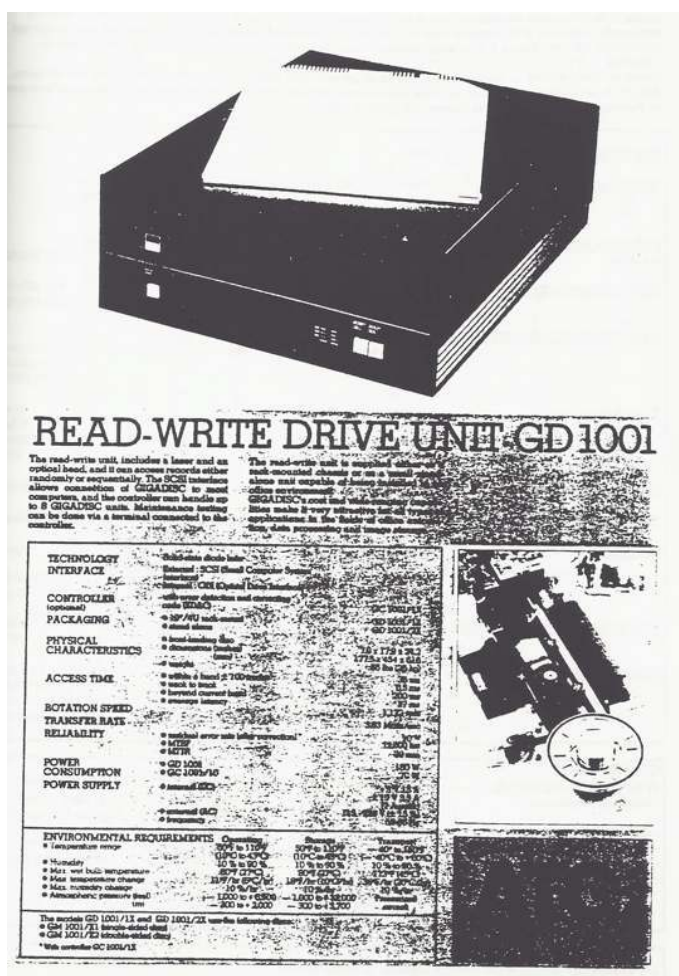

Annexe 2 


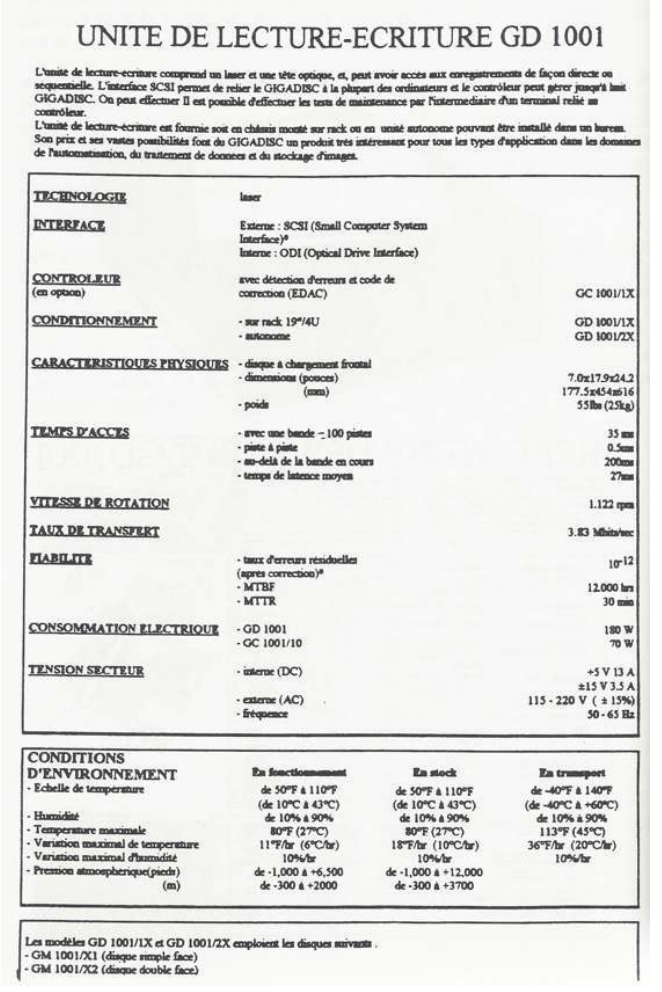

\section{NOTES}

1. Le mot «traduction » est pris au sens large, et englobe interprétation et traduction, sauf si précision.

2. Diplôme universitaire de technologie approfondie, Bac +3 et 4 .

3. Pas toujours le français. Beaucoup de nos étudiants sont étrangers; la langue française n'a été parfois acquise qu'après la langue anglaise. Un cas à envisager: un étudiant anglais pour qui le français est la langue seconde. Ce cas est d'un grand intérêt, car il fait un effet de miroir, et aide à discerner les difficultés d'acquisition de nos étudiants en anglais.

4. Ce qui suit n'est pas exhaustif, tant s'en faut. Nous verrons plus loin un certain nombre de paramètres appartenant à ces différentes rubriques.

5. Profiter de la richesse des matériaux, entre autres, les publicités en langue anglaise, les films de démonstration... Pour l'informatique, un certain nombre d'articles de PC Magazine américain sont traduits dans le $P C$ Expert français, et invitent à une comparaison entre les langues, exercice fructueux.

6. Dès cet instant, on peut, au sein de l'équipe, interpréter les interventions, qui le seront systématiquement, lors des échanges réunissant une partie des équipes, ou l'ensemble de la classe (voir plus loin).

7. Cependant, différences lexicales dues à l'usage américain ou britannique, spécificités commerciales, variantes phonologiques et orthographiques.

8. Même remarque que précédemment sur l'interprétation conseillée.

9. Il est possible d'utiliser deux à trois magnétophones et de les synchroniser : l'un donnant la bande passante, les autres servant aux différentes interprétations. 
10. Ce projet nécessite trois à quatre séances de trois heures, sans compter les mises au point que les étudiants apportent chez eux, les temps d'enregistrement hors de la classe, les photocopies de documents, et le travail sur traitement de textes.

\section{RÉSUMÉS}

Dans le cadre de la formation initiale et continue, en Institut de Technologie, voici la mise à profit de la confrontation de deux domaines : celui de la spécialité technique et celui de la langue anglaise de spécialité, par le biais de la pratique raisonnée de la traduction, qui joue un rôle médiateur, de spécialité à spécialité. L'acquisition du programme d'anglais spécialisé et l'apprentissage des stratégies de la fonction traduisante, dans le domaine de spécialité technique, assurent une mise en perspective professionnelle, à double qualification.

In higher and further education, at the Institute of Technology, there is a fruitful confrontation of two fields, technological studies and English for specific purposes. This is achieved through a reasoning process applied to translation which mediates between the two fields of study. The acquisition of a programme in technical English and training in translating strategies in the students' technical field lead to a professional outlook with double use.

\section{INDEX}

Mots-clés : préprofessionalisme, traduction

Keywords : preprofessionalism, translation

\section{AUTEUR}

\section{SYLVIE LANDOIS-MAYNARD}

IUT de Paris 